\title{
Formulating Assur Kinematic Chains as Projective Extensions of Baranov Trusses
}

\author{
Nicolás Rojas, Federico Thomas* \\ Institut de Robòtica i Informàtica Industrial (CSIC-UPC) \\ Llorens Artigas 4-6, 08028 Barcelona, Spain
}

\begin{abstract}
The real roots of the characteristic polynomial of a planar linkage determine its assembly modes. In this work it is shown how the characteristic polynomial of a Baranov truss derived using a distance-base formulation contains all the necessary and sufficient information for solving the position analysis of the Assur kinematic chains resulting from replacing some of its revolute joints by slider joints. This is a relevant result because it avoids the case-by-case treatment that requires new sets of variable eliminations to obtain the characteristic polynomial of each Assur kinematic chain.
\end{abstract}

Keywords: Position analysis, Assur kinematic chains, Baranov trusses, characteristic polynomial, modular kinematics.

\section{Introduction}

A non-overconstrained linkage with zero-mobility from which an Assur group can be obtained by removing any of its links is defined as an Assur kinematic chain, basic truss [1, 2], or Baranov truss if no slider joints are considered [3]. Hence, a Baranov truss, named after the Russian kinematician G.G. Baranov who first presented the idea of this kind of truss in 1952 [4], corresponds to multiple Assur groups. Baranov, in his seminal paper, presented 3 trusses of 7 links and 26 trusses of 9 links. In 1971, Manolescu and Erdelean identified two additional trusses of 9 links that were missing in the initial classification [5], thus completing the classification of Baranov trusses with up to 4 loops. In 1994, Yang and Yao found that the number of Baranov trusses with 11 links is 239 without explicitly presenting their topology [6]. All Assur kinematic chains can be derived from Baranov trusses by replacing revolute joints by slider joints bearing in mind that loops with only slider joints cannot be considered because they would reduce in one the number of constraints that make an Assur kinematic chain rigid 7]. Therefore, three different 3-link Assur kinematic chains can be derived from the triad - the only Baranov truss with 3 links - 2] [Fig. 1(a)], and ten 5-link Assur kinematic chains from the pentad - the only Baranov truss with 5 links - 8] [Fig. 1(b)].

\footnotetext{
*Corresponding author. Tel.: +34 934015757; fax: +34 934015750.

Email addresses: nrojas@iri.upc.edu (Nicolás Rojas), fthomas@iri.upc.edu (Federico Thomas) Preprint submitted to Mechanism and Machine Theory 


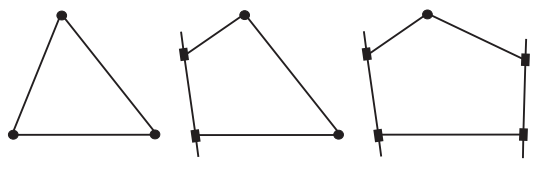

(a)

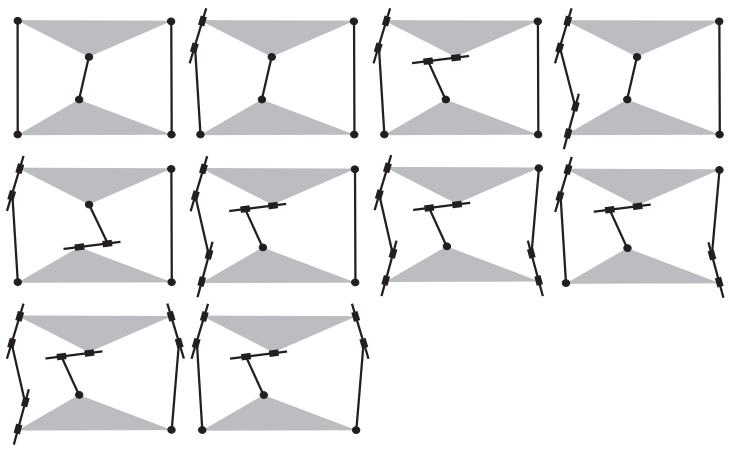

(b)

Figure 1: The three 3-link Assur kinematic chains (a), and the ten 5-link Assur kinematic chains (b).

Closed-form solutions for the position analysis of all Assur kinematic chains with 3 and 5 links have been obtained on an ad hoc basis by several authors. For the three 3 -link Assur kinematic chains, explicit solutions to the position analysis problem can be found, for instance, in [2]. The position analysis problem of the ten 5-link Assur kinematic chains was solved for the first time in closed form by Li and Matthew in 88]. Other solutions for 5 -link Assur kinematic chains have been presented, at least, in [9, 10, 11, 12, 13]. An extensive research on the position analysis of Assur kinematic chains with only revolute joints, i.e. Baranov trusses, has been performed by the kinematics community in the last decades, see [14] and the references therein, to the point that a closed-form solution of a 13-link (6 loop) Baranov truss, without relying on variable eliminations nor trigonometric substitutions, has been recently reported [15]. Beyond 5 links, the closed-form position analysis of some Assur kinematic chains has only been tackled, to our knowledge, by Wohlhart in [16, 17, 18]. In these works, using the Sylvester's elimination method, he successfully solved nine 9-link and one 11-link Assur kinematic chains.

General algorithms indeed exists for the closed-form position analysis of multi-loop planar linkages but they invariably rely on resultant elimination techniques applied to sets of kinematic loop equations. For example, Nielsen and Roth [19], and Wampler [20] presented general methods for the analysis of planar linkages using the Dixon's resultant. Although the uniform treatment of these elimination-based methods of all planar linkages is remarkable, the position analysis of Assur kinematic chains based on them has to be carried out on a case-by-case basis because the required variable eliminations change.

In order to analyze Assur kinematic chains and Baranov trusses in a unified way, one possibility would be to introduce some transformations that would allow us to treat the translations associated with the slider joints as rotations. To this end, at least three options arise:

1. Substituting the slider joints by inversors 21]. Although this would solve the problem, the resulting truss would be, in general, too complicated since one inversor should be introduced for each slider. Moreover, using this kind of substitutions, the analysis of a Baranov truss does not seem to provide any insight on the analysis of their derived Assur kinematic chains. 
2. Adding one extra dimension which would permit to have an origin for the rotations which is lifted outside the $2 \mathrm{D}$ plane. Then, it would be possible to turn translations into rotations. This has to do with the stereographic projection. For example, in the framework of Clifford algebra, translations on a 2D plane are difficult to handle, but if one maps that plane onto the surface of a sphere in $3 \mathrm{D}$, then one can identify the $2 \mathrm{D}$ translations with rotations on the surface of the 3D sphere. Although this approach is mathematically elegant by providing a unified treatment of Baranov trusses and Assur kinematic chains, it seems to give no clear advantage compared to the standard approach based on independent kinematic vector equations because the variables to be eliminated from the set of derived equations also change with the analyzed Assur kinematic chain.

3. Regarding a translational motion as an infinitely small rotation about a point at infinity. It is well-known that a translation in the direction $\left(u_{x}, u_{y}\right)$ may be represented as a rotation about the ideal point given in homogeneous coordinates by $\left(-u_{y}, u_{x}, 0\right)^{T}$. This is probably the most intuitive and simple approach but, depending on the used formulation, it may be difficult to be accommodated. This paper is essentially devoted to show how a coordinate-free formulation based on distances and oriented areas provides a framework within which this idea can be easily applied thus leading to the conclusion that the characteristic polynomials of the Baranov trusses contain all the necessary and sufficient information for solving the position analysis of all derived Assur kinematic chains.

This paper is organized as follows. Section 2 briefly reviews the basics of the used formulation: the concept of bilateration matrix and the idea of deriving closure conditions in terms of this kind of matrices. Then, Section 3 shows how to transform an Assur kinematic chain with slider joints into a Baranov truss with revolute joint centers located at infinity. Section 4 shows through examples how to solve, using the closure condition for a Baranov truss in terms of bilateration matrices, the position analysis of different Assur kinematic chains derived from it. Finally, we conclude in Section 5

\section{Bilateration matrices and closure conditions for Baranov trusses}

In what follows, $P_{i}$ will denote a point, $\overline{P_{i} P_{j}}$ the segment defined by $P_{i}$ and $P_{j}$, and $\triangle P_{i} P_{j} P_{k}$ the triangle defined by $P_{i}, P_{j}$, and $P_{k}$. Moreover, $\mathbf{p}_{i j}=\overrightarrow{P_{i} P_{j}}$ and $s_{i, j}=\left\|\mathbf{p}_{i, j}\right\|^{2}$.

Let us suppose that we want to obtain the point of intersection of two circles centered at $P_{i}$ and $P_{j}$, respectively. Then, according to Fig. 2(a), the result to this problem can be expressed in vector form as:

$$
\mathbf{p}_{i, k}=\mathbf{Z}_{i, j, k} \mathbf{p}_{i, j}
$$

where

$$
\mathbf{Z}_{i, j, k}=\frac{1}{2 s_{i, j}}\left[\begin{array}{cc}
s_{i, j}+s_{i, k}-s_{j, k} & -4 A_{i, j, k} \\
4 A_{i, j, k} & s_{i, j}+s_{i, k}-s_{j, k}
\end{array}\right]
$$

is called a bilateration matrix, and

$$
A_{i, j, k}= \pm \frac{1}{4} \sqrt{\left(s_{i, j}+s_{i, k}+s_{j, k}\right)^{2}-2\left(s_{i, j}^{2}+s_{i, k}^{2}+s_{j, k}^{2}\right)}
$$




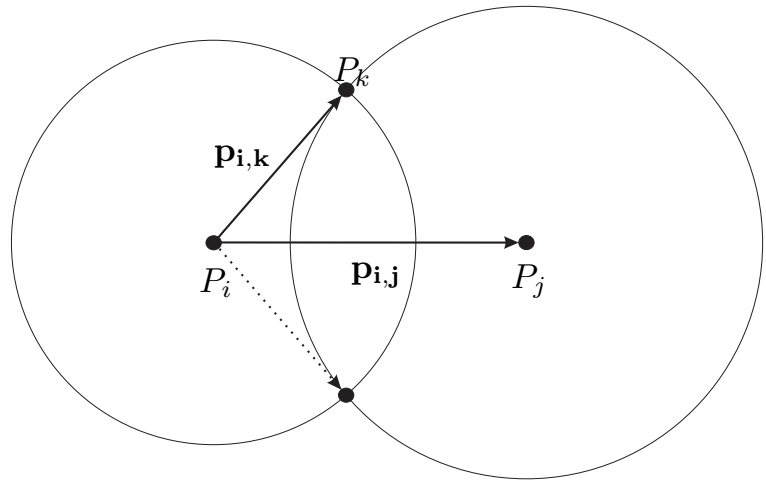

(a)

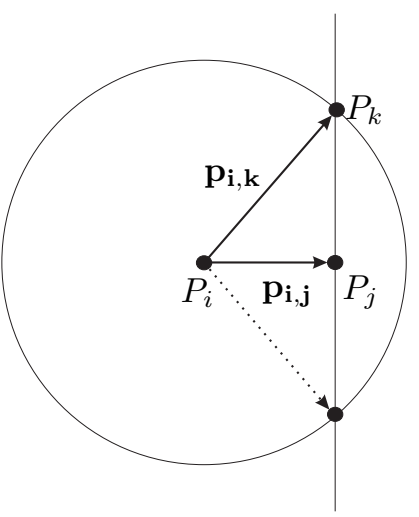

(b)

Figure 2: The intersection of two circles (a), and the intersection of a circle and a line (b) can have up to two possible solutions which can be expressed in vector form using bilateration matrices.

is the oriented area of $\triangle P_{i} P_{j} P_{k}$ which is defined as positive if $P_{k}$ is to the left of the oriented line defined by $\mathbf{p}_{i, j}$, and negative otherwise. The interested reader is addressed to 22] for a derivation of this formula.

Now, let us suppose that we want to obtain the intersection of a circle centered at $P_{i}$ and a line whose nearest point to $P_{i}$ is $P_{j}$. In this case, according to Fig. 2(b), $s_{i, k}=s_{i, j}+s_{j, k}$. Then, substituting this relationship in expression (1), we get

$$
\mathbf{p}_{i, k}=\mathbf{Y}_{i, j, k} \mathbf{p}_{i, j}
$$

where

$$
\mathbf{Y}_{i, j, k}=\frac{1}{s_{i, j}}\left[\begin{array}{cc}
s_{i, j} & -2 A_{i, j, k} \\
2 A_{i, j, k} & s_{i, j}
\end{array}\right]
$$

and

$$
A_{i, j, k}= \pm \frac{1}{2} \sqrt{s_{i, j} s_{i, k}}
$$

is the oriented area of right triangle $\triangle P_{i} P_{j} P_{k}$.

It can checked that, if $\mathbf{v}=\mathbf{Z} \mathbf{w}$, where $\mathbf{Z}$ is a bilateration matrix, then $\|\mathbf{v}\|^{2}=$ $\operatorname{det}(\mathbf{Z})\|\mathbf{w}\|^{2}$. Moreover, it can also be checked that the product of two bilateration matrices is commutative. Then, it is easy to prove that the set of bilateration matrices, i.e., matrices of the form $\left(\begin{array}{cc}a & -b \\ b & a\end{array}\right)$, constitute a commutative group under the product and addition operations.

In those geometric problems where the solution can be constructed using a ruler and a compass only, each point is determined by the intersection of two lines, a line and a circle, or two circles 23]. Thus, bilateration matrices permit to represent the result of these operations in a coordinate-free vector form where the sign of the square roots account for all possible solutions that are generated along the constructive process. The position analysis of a planar linkage is a geometric problem that sometimes can be solved with no further help than a compass and a ruler. The characteristic polynomials of these particular linkages factor into quadratic terms and this is why they are usually called 
quadratically-solvable linkages. Unfortunately, the position analysis of Baranov trusses with more than 3 links cannot be solved using a ruler and a compass but bilateration matrices are still of great practical interest because their use permits to derive necessary and sufficient closure conditions which in most cases directly reduce to a single scalar equation [14]. For example, these conditions for the three 7-link Baranov trusses appear in Fig. 3. They are expressed in terms of an unknown distance (the one that corresponds to the segment shown in dotted line). The different values for this unknown distance that satisfy the corresponding closure condition determine the different assembly modes. Actually, for each of these values, the different assembly modes can be constructed using a ruler and a compass only. Moreover, the characteristic polynomials can be derived from these closure conditions by expanding them, clearing radicals and factorizing the result (see [14, 22] for guidelines on how to perform these operations). Note that this process does not involve any trigonometric substitutions nor variable eliminations.

\section{Projective extensions of Baranov trusses}

Let us suppose that the revolute joint centered at $P_{i}$ in Fig. 廿(a) is replaced by a slider joint as shown in Fig. 4(b) such that $P_{i}$ is split into $P_{i}^{\prime}$ and $P_{i}^{\prime \prime}$. This new joint is placed at fixed orientations with respect to the links connected to them. Once an orientation is assigned to the slider joint axis with respect to its adjacent links, a set of orientation angles can be defined (in this case $\alpha_{j}, \alpha_{k}, \alpha_{l}$, and $\alpha_{m}$ ) and, as a consequence, an oriented distance can be assigned to the points of the links connected by this joint with respect to this axis, as shown in Fig. 4 (c). The sign of the considered distance will be the sign of the sine of the corresponding orientation. This defines a set of new points on the slider axis: those that realize the minimum distance to the considered points (in this case $P_{j}^{\prime}, P_{k}^{\prime}, P_{l}^{\prime}$, and $P_{m}^{\prime}$ ). Note that the slider joint imposes the alignment of all these points but, for the moment, let us suppose that they all are located at the same distance, say $\delta_{i}$, from $P_{i}^{\infty}$ as shown in Fig. 4(d). This would imply that they would lie on a circle but, if $\delta_{i} \rightarrow \infty$, they would again lie on a line as imposed by the slider join. The result of these geometric transformation is a joint whose topology is the same as that of

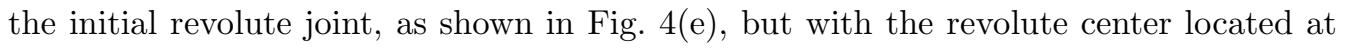
infinity.

It is worth noting that, after the described geometric transformation, it might happen that the orientations of $\triangle P_{i}^{\infty} P_{j} P_{k}$ or $\triangle P_{i}^{\infty} P_{l} P_{m}$ have changed with respect to that of $\triangle P_{i} P_{j} P_{k}$ or $\triangle P_{i} P_{l} P_{m}$, respectively, and this possible sign change has to be taken into account in the closure condition.

Although the described process is conceptually simple, at least two situations arise that required a detailed analysis:

1. when two adjacent revolute joints are replaced by slider joints,

2. when a slider joint replaces a revolute joint acting as an end point of the segment whose distance is used as variable in the closure condition.

Handling these situations is not difficult but requires an explanation that is better understood through examples. This is carried out in the next section where the above geometric transformation is used to solve the position analysis of several Assur kinematic chains derived from the same Baranov truss. 


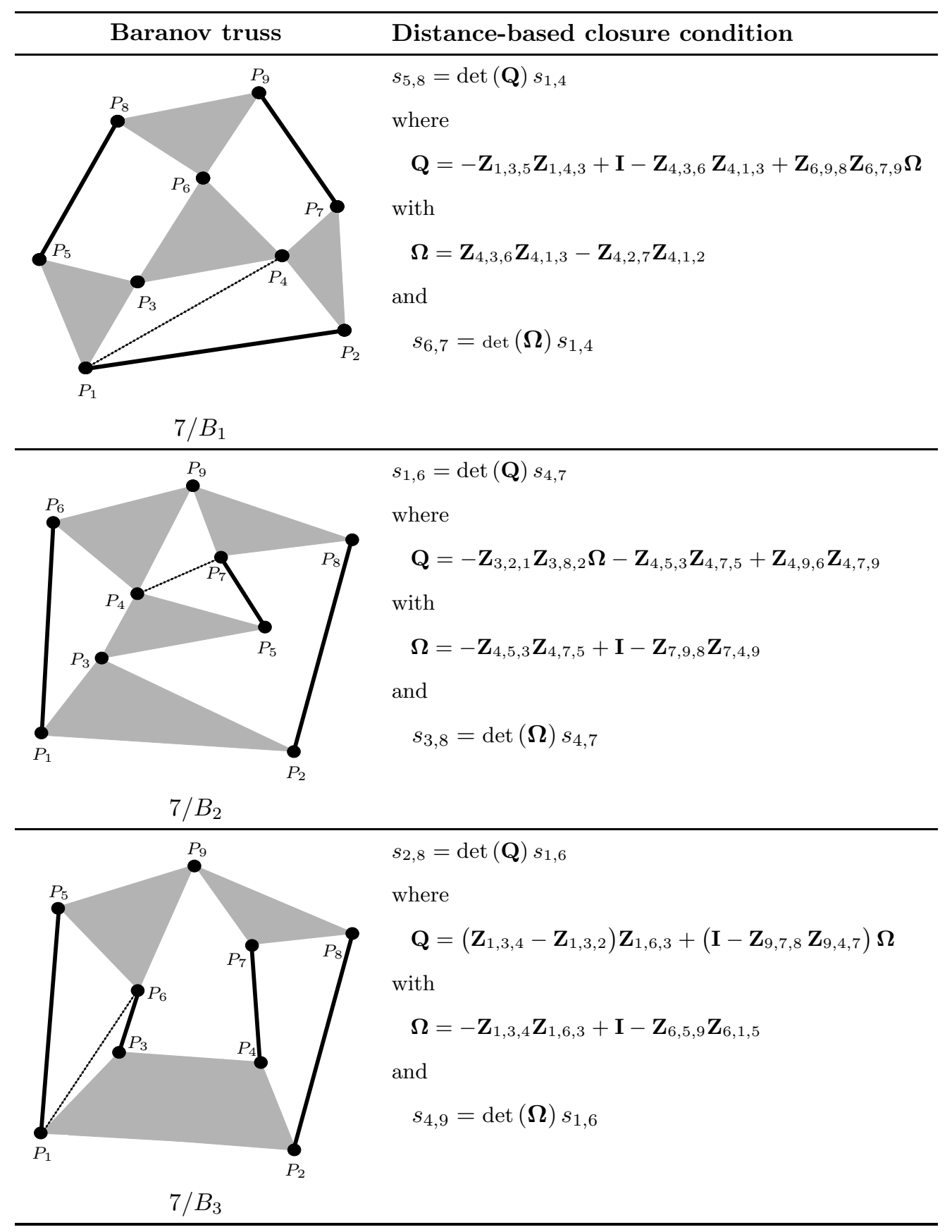

Figure 3: The three 7-link Baranov trusses and their closure conditions in terms of bilateration matrices. The dotted line in each truss represents the unknown distance used as variable in the corresponding closure condition. 


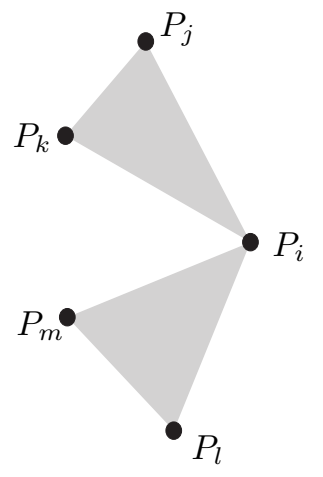

(a)

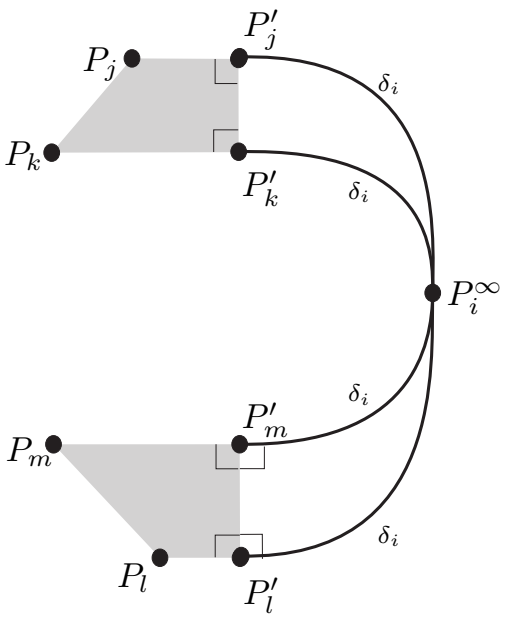

(d)

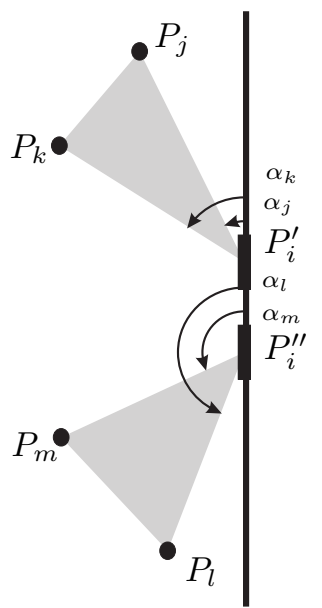

(b)

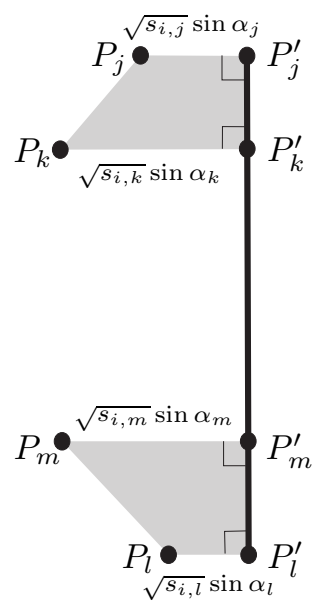

(c)

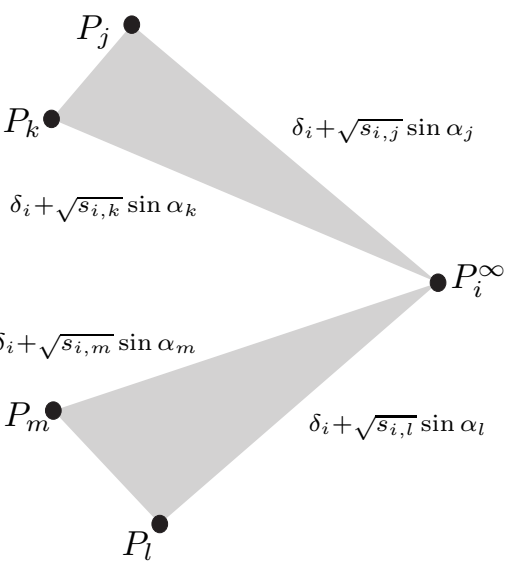

(e)

Figure 4: Geometric transformation that permits a slider joint that replaces a revolute joint be transformed back to a revolute join centered at infinity.

\section{Application to the position analysis of a family of 7-link Assur kinematic chains}

The third Baranov truss in Fig. 3 will be used to exemplify the ideas presented in the previous sections. First, we will show how its characteristic polynomial and, as a consequence, their assembly modes can be derived from its closure condition given in terms of bilateration matrices. Then, we will see the effect of substituting one revolute joint by a slider joint — as shown in Fig. 6(a) - on this closure condition using the geometric transformations described in the previous section. This analysis leaves the 
way paved for the analysis of the Assur kinematic chains shown in Fig. 6(c), in which two adjacent revolute joints have been replaced by slider joints, and that shown in Fig. 9 , in which one of the joints defining one of the end-points of the segment whose length is used as variable in the closure condition is replaced by a slider joint.

\subsection{The assembly modes of the $7 / B_{3}$ Baranov truss}

Let us consider the $7 / B_{3}$ truss in Fig. 3. It has three independent kinematic loops and nine joints. The reader is addressed to [22, 24] for a detailed analysis of this truss. Its distance-based closure condition, according to Fig. 3, is:

$$
s_{2,8}=\operatorname{det}(\mathbf{Q}) s_{1,6},
$$

where

$$
\mathbf{Q}=\left(\mathbf{Z}_{1,3,4}-\mathbf{Z}_{1,3,2}\right) \mathbf{Z}_{1,6,3}+\left(\mathbf{I}-\mathbf{Z}_{9,7,8} \mathbf{Z}_{9,4,7}\right) \boldsymbol{\Omega}
$$

with $\boldsymbol{\Omega}=-\mathbf{Z}_{1,3,4} \mathbf{Z}_{1,6,3}+\mathbf{I}-\mathbf{Z}_{6,5,9} \mathbf{Z}_{6,1,5}$ and $s_{4,9}=\operatorname{det}(\boldsymbol{\Omega})$. This equation expresses the set of values of $s_{1,6}$ compatible with all links side lengths and the signs of the oriented areas of the triangles $\triangle P_{5} P_{6} P_{9}, \triangle P_{7} P_{8} P_{9}, \triangle P_{1} P_{2} P_{3}$ and $\triangle P_{2} P_{3} P_{4}$. Once the dimensions of the truss links have been substituted in equation (6) and the result expanded, a scalar radical equation in function of the unknown squared distance $s_{1,6}$ is obtained which can be solved using, for example, a Newton interval method. Alternatively, a polynomial representation can be derived. For example, according to the notation used in Fig. 6, let us set $s_{1,2}=49, s_{1,3}=13, s_{1,4}=29, s_{1,5}=101, s_{2,3}=34, s_{2,4}=8, s_{2,8}=82, s_{3,4}=10$, $s_{3,6}=36, s_{4,7}=52, s_{5,6}=10, s_{5,9}=29, s_{6,9}=13, s_{7,8}=10, s_{7,9}=34$, and $s_{8,9}=20$. Substituting these values in equation (6), expanding it, clearing radicals, and factorizing the result, we obtain the characteristic polynomial:

$$
\begin{aligned}
& s_{1,6}^{18}-1146.0063 s_{1,6}^{17}+6.175410^{5} s_{1,6}^{16}-2.075510^{8} s_{1,6}^{15}+4.868410^{10} s_{1,6}^{14} \\
& -8.451510^{12} s_{1,6}^{13}+1.123910^{15} s_{1,6}^{12}-1.169310^{17} s_{1,6}^{11}+9.636910^{18} s_{1,6}^{10} \\
& -6.330710^{20} s_{1,6}^{9}+3.318710^{22} s_{1,6}^{8}-1.383210^{24} s_{1,6}^{7}+4.543210^{25} s_{1,6}^{6} \\
& -1.158010^{27} s_{1,6}^{5}+2.236010^{28} s_{1,6}^{4}-3.148910^{29} s_{1,6}^{3}+3.038210^{30} s_{1,6}^{2} \\
& -1.787710^{31} s_{1,6}+4.822610^{31} .
\end{aligned}
$$

The real roots of this polynomial are 88.5700 and 90.8322 . The corresponding assembly modes, for the case in which the ground link is the quaternary link with points located at $P_{1}=(1,0)^{T}, P_{2}=(8,0)^{T}, P_{3}=(3,3)^{T}$, and $P_{4}=(6,2)^{T}$, appear in Fig. 5.

Next, we will see the effect of replacing a revolute joint by a slider joint on this truss.

\subsection{Replacing one revolute joint}

When the revolute joint centered at $P_{9}$ in the above Baranov truss is replaced by a slider joint as indicated in Fig. 6(a), an Assur kinematic chain is obtained. In accordance with the notation used in Fig. 6(b), the distance-based closure condition of this new linkage can be obtained, as explained in Section [3, by setting in equation (6): 

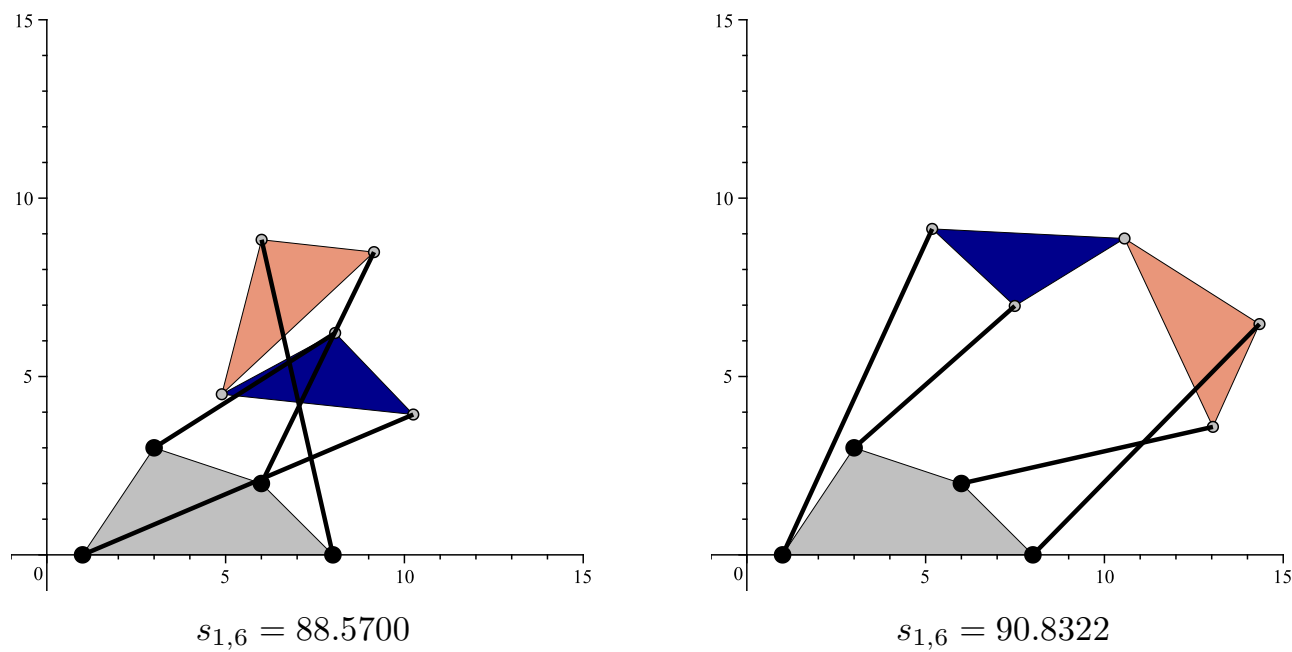

Figure 5: The assembly modes of the $7 / B_{3}$ Baranov truss used as reference truss.

$$
\begin{aligned}
& s_{5,9}=\left(\delta_{1}+\sqrt{s_{5,9^{\prime}}} \sin \alpha_{1}\right)^{2} \\
& s_{6,9}=\left(\delta_{1}+\sqrt{s_{6,9^{\prime}}} \sin \alpha_{2}\right)^{2} \\
& s_{7,9}=\left(\delta_{1}+\sqrt{s_{7,9^{\prime \prime}}} \sin \alpha_{3}\right)^{2} \\
& s_{8,9}=\left(\delta_{1}+\sqrt{s_{8,9^{\prime \prime}}} \sin \alpha_{4}\right)^{2}
\end{aligned}
$$

and taking the limit $\delta_{1} \rightarrow \infty$. That is,

$$
\lim _{\delta_{1} \rightarrow \infty}\left(\operatorname{det}(\mathbf{Q}) s_{1,6}-s_{2,8}\right) \mid \begin{aligned}
& s_{5,9}=\left(\delta_{1}+\sqrt{s_{5,9^{\prime}}} \sin \alpha_{1}\right)^{2} \\
& s_{6,9}=\left(\delta_{1}+\sqrt{s_{6,9^{\prime}}} \sin \alpha_{2}\right)^{2} \\
& s_{7,9}=\left(\delta_{1}+\sqrt{s_{7,9^{\prime \prime}}} \sin \alpha_{3}\right)^{2} \\
& s_{8,9}=\left(\delta_{1}+\sqrt{s_{8,9^{\prime \prime}}} \sin \alpha_{4}\right)^{2}
\end{aligned} .
$$

Note that the expression $\operatorname{det}(\mathbf{Q}) s_{1,6}-s_{2,8}$, after the substitutions, can be written as a polynomial in $\delta_{1}$. Therefore, the above limit can be expressed as:

$$
\lim _{\delta_{1} \rightarrow \infty} \sum_{i=0}^{n} \phi_{i}\left(s_{1,6}\right) \delta_{1}^{i} \mid \begin{aligned}
& s_{5,9}=\left(\delta_{1}+\sqrt{s_{5,9^{\prime}}} \sin \alpha_{1}\right)^{2} \\
& s_{6,9}=\left(\delta_{1}+\sqrt{s_{6,9^{\prime}}} \sin \alpha_{2}\right)^{2} \\
& s_{7,9}=\left(\delta_{1}+\sqrt{s_{7,9^{\prime \prime}}} \sin \alpha_{3}\right)^{2} \\
& s_{8,9}=\left(\delta_{1}+\sqrt{s_{8,9^{\prime \prime}}} \sin \alpha_{4}\right)^{2} .
\end{aligned}
$$

Then, we conclude that, for this limit to be zero, $\phi_{n}\left(s_{1,6}\right)=0$. In other words, the new distance-based closure condition is $\phi_{n}\left(s_{1,6}\right)=0$. 


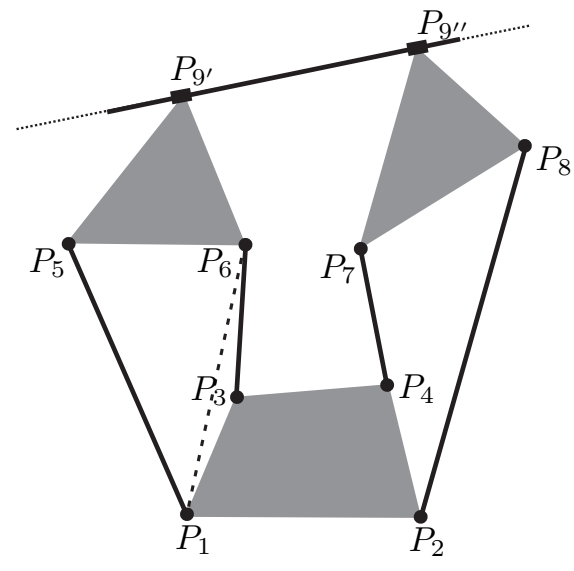

(a)

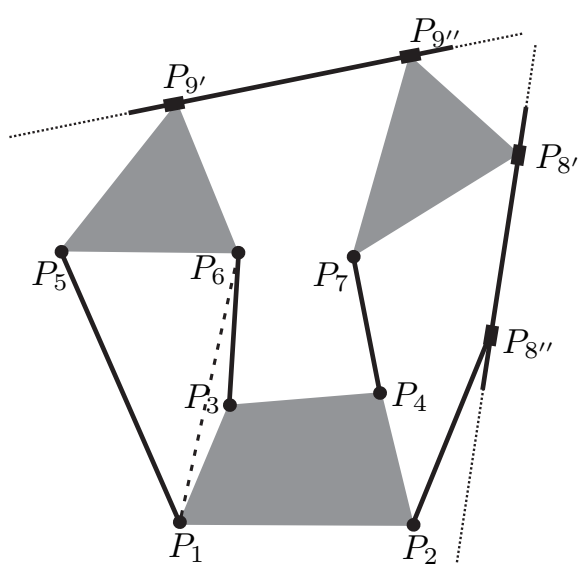

(c)

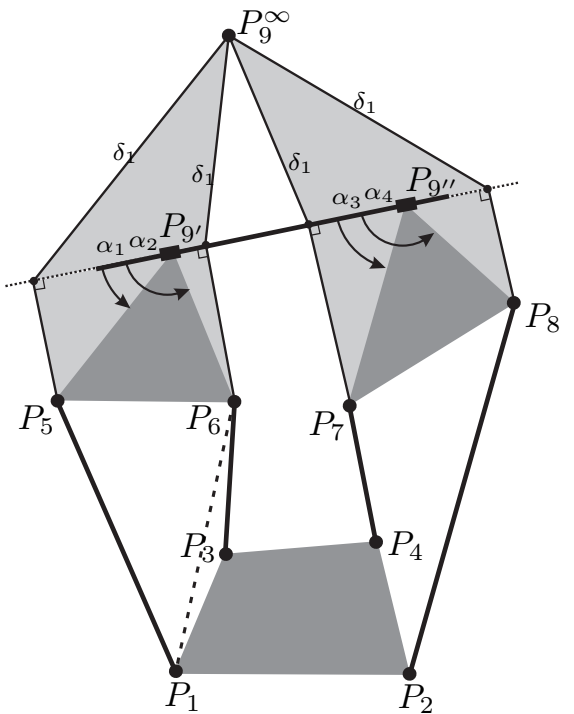

(b)

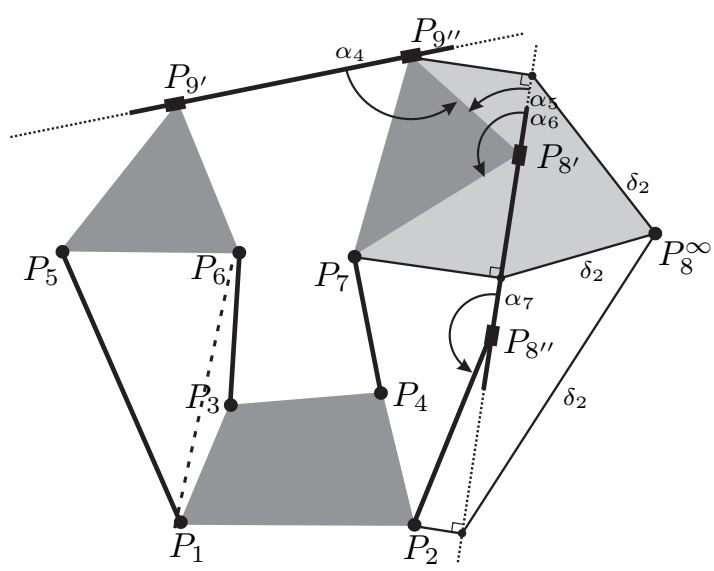

(d)

Figure 6: The distance-based closure condition of the 7-link Assur kinematic chain with one slider joint in (a) can be obtained by properly substituting the square lengths $s_{5,9}, s_{6,9}, s_{7,9}$, and $s_{8,9}$ in the original closure condition and taking the limit $\delta_{1} \rightarrow \infty(\mathrm{b})$. The distance-based closure condition of the 7-link Assur kinematic chain with two slider joints (c) can be computed by properly substituting $\alpha_{4}, s_{8,9^{\prime \prime}}$, $s_{7,8}$, and $s_{2,8}$ in the closure condition obtained for the 7-link Assur kinematic chain with one slider joint and taking the limit $\delta_{2} \rightarrow \infty$ (d). 
Now, let us suppose that all link dimensions remain unaltered, that is, $s_{5,9^{\prime}}=s_{5,9}$, $s_{6,9^{\prime}}=s_{6,9}, s_{7,9^{\prime \prime}}=s_{7,9}$, and $s_{8,9^{\prime \prime}}=s_{8,9}$, and the orientation of the slider joint is fixed with respect to its adjacent links such that $\alpha_{1}=\pi-\arctan \frac{5}{2}$ and $\alpha_{3}=2 \pi-$ $\arctan \frac{5}{3}$. Then, given the orientations of $\triangle P_{5} P_{6} P_{9^{\prime}}$ and $\triangle P_{7} P_{8} P_{9^{\prime \prime}}$, it turns out that $\alpha_{2}=\pi-\arctan \frac{2}{3}$ and $\alpha_{4}=2 \pi-\arctan \frac{1}{2}$. Substituting these values in equation (8), expanding it, computing the limit, clearing radicals, and factorizing the result, we obtain the characteristic polynomial

$$
\begin{aligned}
& s_{1,6}^{16}-1088.1889 s_{1,6}^{15}+5.562910^{5} s_{1,6}^{14}-1.775910^{8} s_{1,6}^{13}+3.968710^{10} s_{1,6}^{12} \\
& -6.591110^{12} s_{1,6}^{11}+8.426310^{14} s_{1,6}^{10}-8.474810^{16} s_{1,6}^{9}+6.797910^{18} s_{1,6}^{8} \\
& -4.384210^{20} s_{1,6}^{7}+2.280210^{22} s_{1,6}^{6}-9.522810^{23} s_{1,6}^{5}+3.142610^{25} s_{1,6}^{4} \\
& -7.912110^{26} s_{1,6}^{3}+1.423310^{28} s_{1,6}^{2}-1.616910^{29} s_{1,6}+8.639010^{29}
\end{aligned}
$$

The real roots of this polynomial are 49.5561, 50.1965, 50.6270, and 85.0000. These new assembly modes appear in Fig. 7 .

\subsection{Replacing two adjacent revolute joints}

Now, let us suppose that the revolute joint centered at $P_{8}$ is also replaced by a slider joint as shown in Fig. 6(c). Then, according to the notation used in Fig. 6(d), the closure condition for the resulting 7-link Assur kinematic chain is obtained by setting in the expression resulting from (8)

$$
\begin{aligned}
\alpha_{4} & =\alpha_{4}-\alpha_{5}+\frac{\pi}{2} \\
s_{8,9^{\prime \prime}} & =\left(\delta_{2}+\sqrt{s_{8^{\prime}, 9^{\prime \prime}}} \sin \alpha_{5}\right)^{2} \\
s_{7,8} & =\left(\delta_{2}+\sqrt{s_{7,8^{\prime}}} \sin \alpha_{6}\right)^{2} \\
s_{2,8} & =\left(\delta_{2}+\sqrt{s_{3,8^{\prime \prime}}} \sin \alpha_{7}\right)^{2}
\end{aligned}
$$

and taking the limit for $\delta_{2} \rightarrow \infty$. That is,

$$
\lim _{\delta_{2} \rightarrow \infty}\left(\lim _{\delta_{1} \rightarrow \infty}\left(\operatorname{det}(\mathbf{Q}) s_{1,6}-s_{2,8}\right)\right) \mid \begin{aligned}
& s_{5,9}=\left(\delta_{1}+\sqrt{s_{5,9^{\prime}}} \sin \alpha_{1}\right)^{2} \\
& s_{6,9}=\left(\delta_{1}+\sqrt{s_{6,9^{\prime}}} \sin \alpha_{2}\right)^{2} \\
& s_{7,9}=\left(\delta_{1}+\sqrt{s_{7,9^{\prime \prime}}} \sin \alpha_{3}\right)^{2} \\
& s_{8,9}=\left(\delta_{1}+\left(\delta_{2}+\sqrt{s_{8^{\prime}, 9^{\prime \prime}}} \sin \alpha_{5}\right) \cos \left(\alpha_{4}-\alpha_{5}\right)\right)^{2} \\
& s_{7,8}=\left(\delta_{2}+\sqrt{s_{7,8^{\prime}}} \sin \alpha_{6}\right)^{2} \\
& s_{2,8}=\left(\delta_{2}+\sqrt{s_{3,8^{\prime \prime}}} \sin \alpha_{7}\right)^{2}
\end{aligned}
$$

The important point here is to realize that $\alpha_{4}$, which was introduced in the first set of substitutions, has to be substituted in the second set of substitutions. Thus, both sets of substitutions are not independent. This only happens when replacing two neighboring revolute joints. Note that, if we would have proceeded in the reverse order, the substitution for the squared distance $s_{8,9}$ would have been different. 


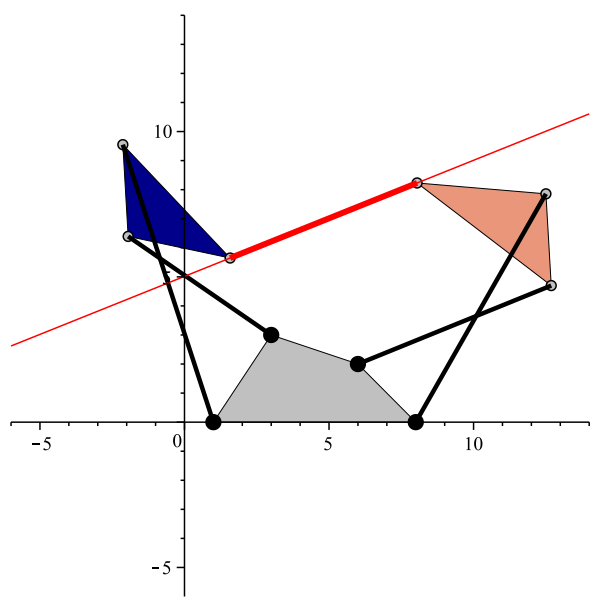

$s_{1,6}=49.5561$

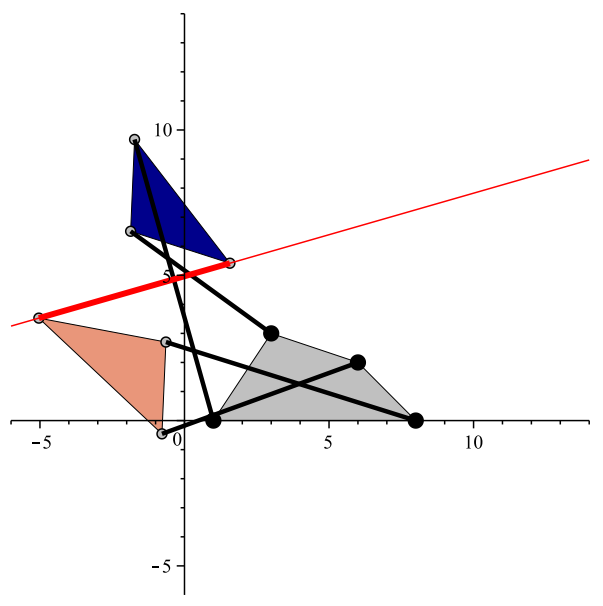

$s_{1,6}=50.6270$

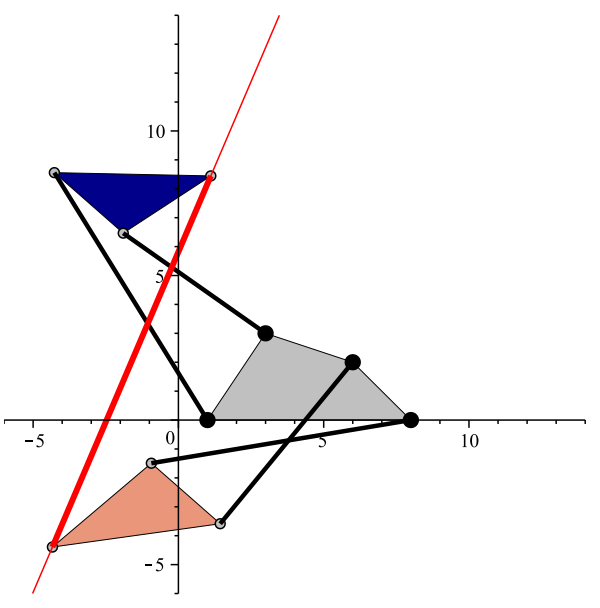

$s_{1,6}=50.1965$

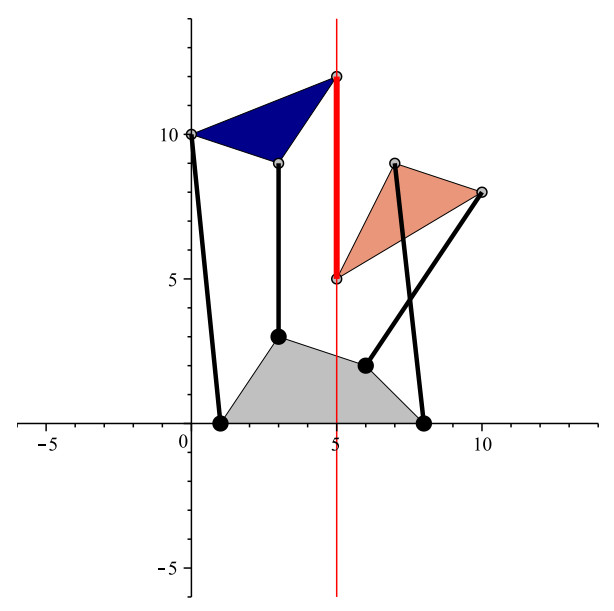

$s_{1,6}=85.0000$

Figure 7: The assembly modes of the analyzed Assur kinematic chain with one slider joint.

According to the notation used in Fig. 6(d), let us again suppose that the dimensions of all links remain unaltered, that is, $s_{2,8^{\prime \prime}}=s_{2,8}, s_{5,9^{\prime}}=s_{5,9}, s_{6,9^{\prime}}=s_{6,9}, s_{7,8^{\prime}}=s_{7,8}$, $s_{7,9^{\prime \prime}}=s_{7,9}$, and $s_{8^{\prime}, 9^{\prime \prime}}=s_{8,9}$, and the orientation of the second slider joint is fixed with respect to its adjacent links such that $\alpha_{5}=\pi-\arctan 3$ and $\alpha_{7}=\pi-\arctan 4$. Then, given the orientation of $\triangle P_{7} P_{8^{\prime}} P_{9^{\prime \prime}}$, it turns out that $\alpha_{6}=\pi+\arctan \frac{1}{2}$. Substituting these values in the expression resulting from (9), expanding it, computing the limit, and clearing radicals, we obtain the following characteristic polynomial: 


$$
\begin{aligned}
& s_{1,6}^{12}-801.1113 s_{1,6}^{11}+2.871610^{5} s_{1,6}^{10}-6.097010^{7} s_{1,6}^{9}+8.562110^{9} s_{1,6}^{8} \\
& -8.421110^{11} s_{1,6}^{7}+6.000410^{13} s_{1,6}^{6}-3.164510^{15} s_{1,6}^{5}+1.249610^{17} s_{1,6}^{4} \\
& -3.685410^{18} s_{1,6}^{3}+7.868010^{19} s_{1,6}^{2}-1.106710^{21} s_{1,6}+7.776510^{21} .
\end{aligned}
$$

The real roots of this polynomial are $48.1037,85.0000,88.2000$, and 88.5483 . The corresponding assembly modes appear in Fig. 8

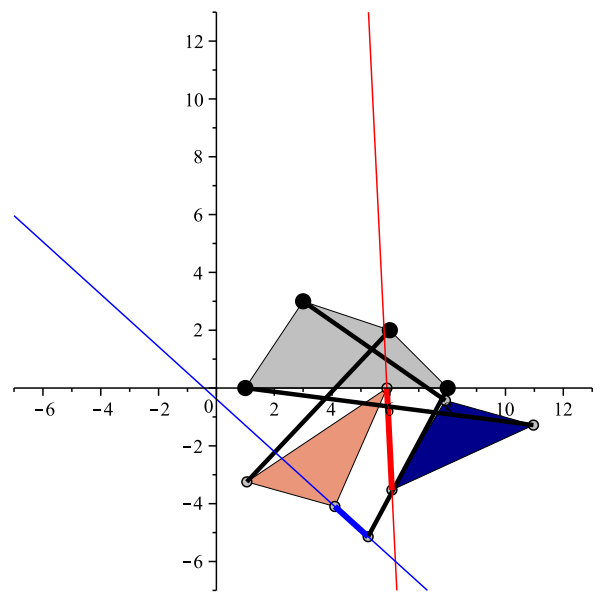

$s_{1,6}=48.1037$

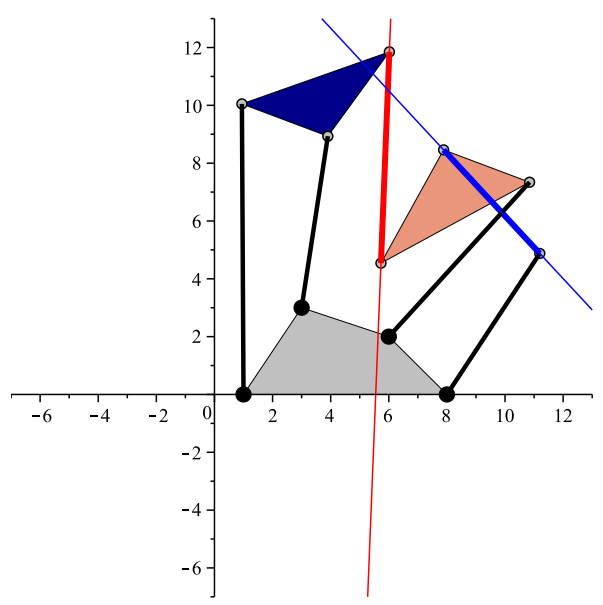

$s_{1,6}=88.2000$

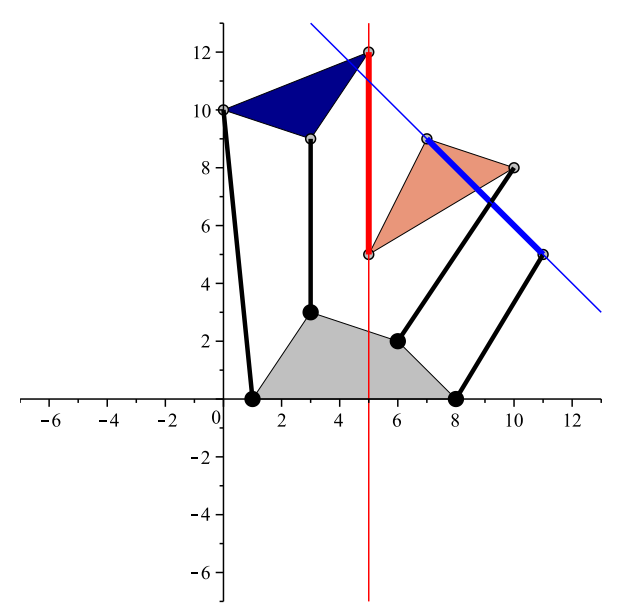

$s_{1,6}=85.0000$

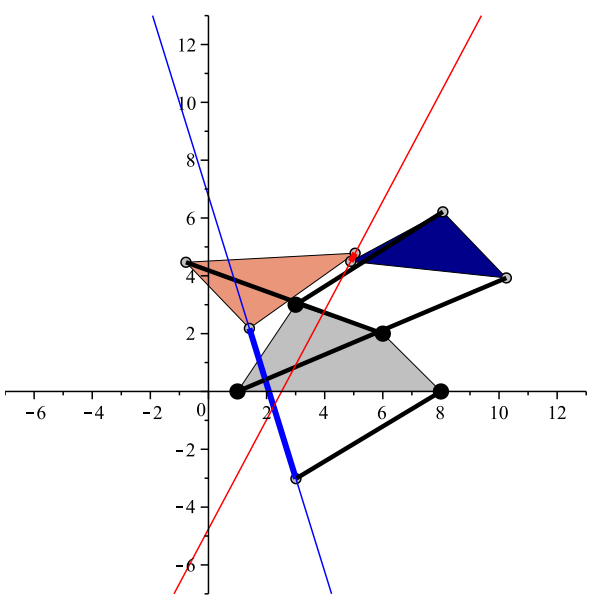

$s_{1,6}=88.5483$

Figure 8: The assembly modes of the analyzed Assur kinematic chain with two slider joints.

\subsection{Replacing one revolute joint involved in the definition of the variable distance}

According to Fig. 9] let us now suppose that the revolute joint centered at $P_{1}$ is replaced by a slider joint in the original Baranov truss. In this case, the substitutions to 
be performed in expression (6) are:

$$
\begin{aligned}
& s_{2,1}=\left(\delta_{3}+\sqrt{s_{2,1^{\prime}}} \sin \alpha_{1}\right)^{2} \\
& s_{4,1}=\left(\delta_{3}+\sqrt{s_{4,1^{\prime}}} \sin \alpha_{2}\right)^{2} \\
& s_{3,1}=\left(\delta_{3}+\sqrt{s_{3,1^{\prime}}} \sin \alpha_{3}\right)^{2} \\
& s_{5,1}=\left(\delta_{3}+\sqrt{s_{5,1^{\prime}}} \sin \alpha_{4}\right)^{2} \\
& s_{6,1}=\left(\delta_{3}+t\right)^{2}
\end{aligned}
$$

The new closure condition, obtained after computing the limit for $\delta_{3} \rightarrow \infty$, depends on a new variable, t, the oriented distance between $P_{6}$ and the slider joint axis.

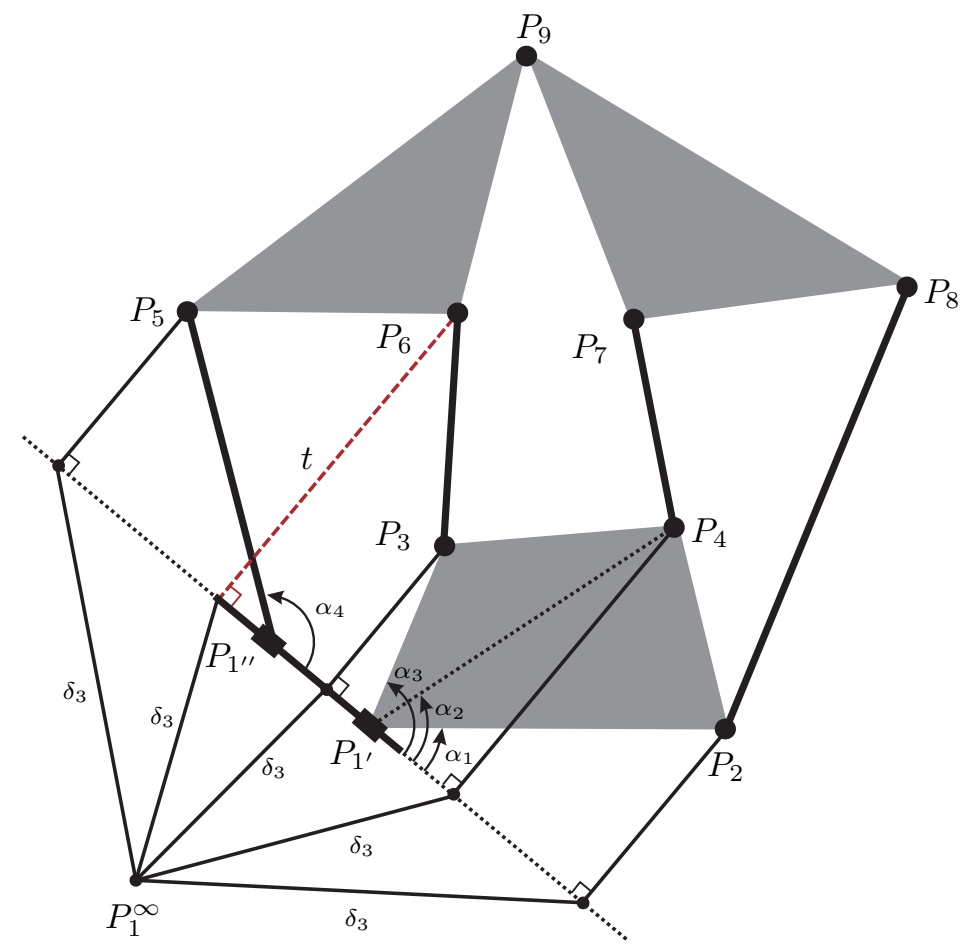

Figure 9: Example in which a slider replaces the revolute join involved in the definition of the distance in which the closure condition of the truss is expressed.

According to the notation used in Fig. 9, let us suppose as above that the dimension of all the original truss links remain unaltered after this substitution, that is, $s_{1^{\prime}, 2}=s_{1,2}$, $s_{1^{\prime}, 3}=s_{1,3}, s_{1^{\prime}, 4}=s_{1,4}, s_{1^{\prime \prime}, 5}=s_{1,5}$, and the orientation of the introduced slider joint axis with respect to its adjacent links is given by $\alpha_{1}=0$ and $\alpha_{4}=\arctan 10$. Then, given the orientations of $\triangle P_{1^{\prime}} P_{2} P_{4}$ and $\triangle P_{1^{\prime}} P_{4} P_{3}$, it turns out that $\alpha_{2}=\arctan \frac{2}{5}$ and $\alpha_{3}=\arctan \frac{3}{2}$. Finally, performing the substitutions given in (10) in equation (6), expanding the result, computing the limit, and clearing radicals, we obtain the characteristic polynomial: 


$$
\begin{aligned}
& t^{18}-99.9226 t^{17}+4616.5154 t^{16}-1.311110^{5} t^{15}+2.570310^{6} t^{14} \\
& -3.707110^{7} t^{13}+4.097510^{8} t^{12}-3.570710^{9} t^{11}+2.503310^{10} t^{10} \\
& -1.432210^{11} t^{9}+6.764910^{11} t^{8}-2.675210^{12} t^{7}+9.066710^{12} t^{6} \\
& -2.728710^{13} t^{5}+7.530010^{13} t^{4}-1.881910^{14} t^{3}+3.883710^{14} t^{2} \\
& -5.535910^{14} t+3.867110^{14}
\end{aligned}
$$

The real roots of this polynomial are 7.4867 and 8.7825 . The corresponding assembly modes appear in Fig. 10,
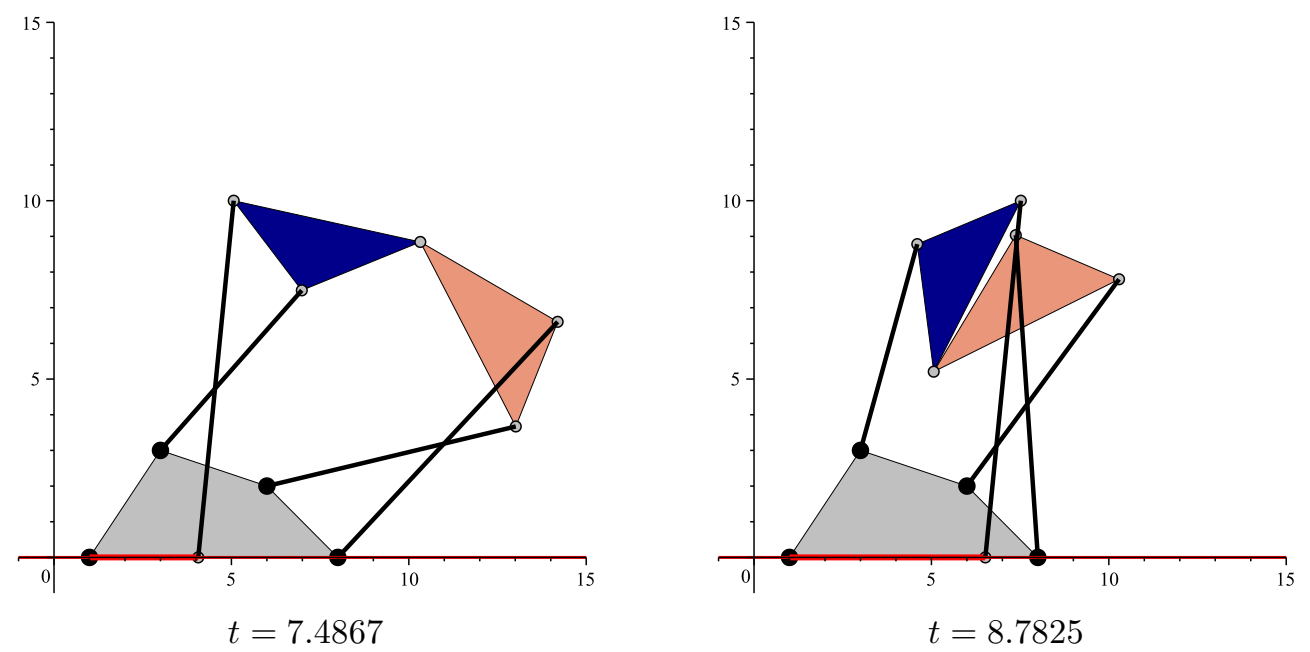

Figure 10: The assembly modes of the 7-link Assur kinematic chain whose closure condition is expressed in terms of the oriented distance between a revolute joint and the slider joint axis.

Note that the case in which two slider joints replace the two revolute joints defining the endpoints of the segment whose length is used as variable in the closure condition can always be avoided because all kinematic loops of an Assur kinematic chain contain at least one revolute joint.

The coefficients of the characteristic polynomials for all the presented examples had to be computed in exact rational arithmetic. Otherwise, numerical problems make impracticable the correct computation of its roots. Although these coefficients have been given in floating point arithmetic for saving space, they could be of interest for comparison and reproducibility purposes.

\section{Conclusion}

Various approaches adopted in different fields, such as linkage analysis, robot kinematics and variational CAD systems, rely on modular methods based on the common idea that complex systems can be decomposed into modules that can be analyzed separately. These modules, called in the field of linkage analysis Assur kinematic chains, 
reduce to Baranov trusses if only revolute joints are considered. In this paper, we have shown how all Assur kinematic chains can be seen as projective extensions of Baranov trusses, that is, Baranov trusses with revolute joint centers located at infinity and how this fact can be easily accommodated in a distance-based formulation. This result leads to the conclusion that the closure conditions for the Baranov trusses thus formulated can be directly used to solve the position analysis of all Assur kinematic chains derived from them. Since the distance-based closure conditions for all cataloged Baranov trusses have recently been published [14], the position analysis of all derived Assur kinematics chains can be carried out without having to perform new sets of variable eliminations, as it is the usual practice when deriving characteristic polynomials from sets of independent vector loop equations.

\section{References}

[1] C. Galletti, A note on modular approaches to planar linkage kinematic analysis, Mechanism and Machine Theory 21 (5) (1986) 385 - 391.

[2] E. Ceresole, P. Fanghella, C. Galletti, Assur's groups, AKCs, basic trusses, SOCs, etc.: Modular kinematics of planar linkages, 96-DETC/MECH-1027, in: Proceedings of the ASME 1996 International Design Engineering Technical Conferences and Computers in Engineering Conference, 1996.

[3] E. Peisach, On Assur groups, Baranov trusses, Grübler chains, planar linkages and on their structural (number) synthesis, in: The 22th Working Meeting of the IFToMM Permanent Commission for Standardization of Terminology, LaMCoS - INSA de Lyon, Villeurbanne, France, 2008, pp. 33 $-41$.

[4] G. Baranov, Classification, formation, kinematics, and kinetostatics of mechanisms with pairs of the first kind (in russian), in: Proceedings of Seminar on the Theory of Machines and Mechanisms, Moscow, Vol. 2, 1952, pp. $15-39$.

[5] N. Manolescu, T. Erdelean, La determination des fermés Baranov avec e=9 elements en utilisant la méthode de graphisation inverse, paper D-12, in: Proceedings of the 3rd IFToMM World Congress on the Theory of Machines and Mechanisms, September, Kupari, Yugoslavia, Vol. D, 1971, pp. 177 $-188$.

[6] T. Yang, F. Yao, Topological characteristics and automatic generation of structural synthesis of planar mechanisms based on the ordered single-opened-chains, in: Proceedings of ASME 1994 Mechanisms Conference, Vol. 70, 1994, pp. $67-74$.

[7] A. Klein, Kinematics of Machinery, McGraw-Hill Book Company, Inc., New York, 1917.

[8] S. Li, G. Matthew, Closed form kinematic analysis of planar Assur II groups, in: Proceedings of the 7th IFToMM World Congress on the Theory of Machines and Mechanisms, September, Sevilla, Spain, Vol. 1, 1987, pp. $141-145$.

[9] S. Mitsi, K.-D. Bouzakis, G. Mansour, I. Popescu, Position analysis in polynomial form of planar mechanisms with Assur groups of class 3 including revolute and prismatic joints, Mechanism and Machine Theory 38 (12) (2003) 1325 - 1344.

[10] S. Mitsi, K.-D. Bouzakis, G. Mansour, Position analysis in polynomial form of planar mechanism with an Assur group of class 4 including one prismatic joint, Mechanism and Machine Theory 39 (3) (2004) $237-245$.

[11] M. Hayes, P. Zsombor-Murray, C. Chen, Unified kinematic analysis of general planar parallel manipulators, Journal of Mechanical Design 126 (5) (2004) 866-874.

[12] W.-Y. Chung, The position analysis of Assur kinematic chain with five links, Mechanism and Machine Theory 40 (9) (2005) 1015 - 1029.

[13] S. Mitsi, K.-D. Bouzakis, G. Mansour, I. Popescu, Position analysis in polynomial form of classthree Assur groups with two or three prismatic joints, Mechanism and Machine Theory 43 (11) (2008) $1401-1415$.

[14] N. Rojas, F. Thomas, On closed-form solutions to the position analysis of Baranov trusses, Mechanism and Machine Theory 50 (2012) $179-196$.

[15] N. Rojas, F. Thomas, Closed-form solution to the position analysis of Watt-Baranov trusses using the bilateration method, Journal of Mechanisms and Robotics 3 (3) (2011) 031001. 
[16] K. Wohlhart, Robots based on Assur group A(3.5), in: J. Lenarcic, P. Wenger (Eds.), Advances in Robot Kinematics: Analysis and Design, Springer Netherlands, 2008, pp. 165-175.

[17] K. Wohlhart, Position analyses of open normal Assur groups A(3.6), in: ASME/IFToMM International Conference on Reconfigurable Mechanisms and Robots, 2009, pp. 88 - 94 .

[18] K. Wohlhart, Position analyses of normal quadrilateral Assur groups, Mechanism and Machine Theory 45 (9) (2010) $1367-1384$.

[19] J. Nielsen, B. Roth, Solving the input/output problem for planar mechanisms, Journal of Mechanical Design 121 (2) (1999) 206-211.

[20] C. Wampler, Solving the kinematics of planar mechanisms by Dixon determinant and a complexplane formulation, Journal of Mechanical Design 123 (3) (2001) 382-387.

[21] E. Dijksman, Motion Geometry of Mechanisms, Chapter 8, Inversors, Cambridge University Press, 1976

[22] N. Rojas, F. Thomas, Distance-based position analysis of the three seven-link Assur kinematic chains, Mechanism and Machine Theory 46 (2) (2011) 112 - 126.

[23] N. Kazarinoff, Ruler and the Round: Classic Problems in Geometric Constructions, Dover Publications, 2003.

[24] C. Innocenti, Polynomial solution to the position analysis of the 7-link Assur kinematic chain with one quaternary link, Mechanism and Machine Theory 30 (8) (1995) 1295 - 1303. 